\title{
Multi-scale Simulations
}

\section{Yam Chi Yung* \\ Beijing Computational Science Research Center, China}

Since the dawn of quantum theory in $20^{\text {th }}$ century, scientists had been utilizing quantum mechanics to predict and explain different phenomena in microscopic world. In the quantum world, electrons, nuclei, light and elementary particles are pictured as both particle- and wave-like. The peculiar nature of quantum mechanics has forced scientist to rethink the nature of matters at the deepest level. While quantum mechanics enables accurate description of different phenomena in microscopic level, the exact solution of Schrödinger equation for many electrons system is intractable. This attributes to the poor scaling of quantum mechanical methods where the computational time cost grows exponentially with number of electrons. As Paul Dirac said in 1929, "The underlying physical laws necessary for the mathematical theory of a large part of physics and the whole of chemistry are thus completely known, and the difficulty is only that the exact application of these laws leads to equations much too complicated to be soluble. It therefore becomes desirable that approximate practical methods of applying quantum mechanics should be developed, which can lead to an explanation of the main features of complex atomic systems without too much computation".

Today, researchers including chemists, physicists and engineers are routinely using quantum mechanical methods through computer software packages to carry their research work. Different levels of approximations are exploited in these methods. Generally, these approximations can be divided into two types: simplified treatment of electron-electron interactions and limitation of electronic wavefunction to the space of linear combinations of a finite basis set. Typical methods include tight-binding method, Hartree-Fock method, density functional theory (DFT), many body perturbation theory, coupled cluster (CC) method and configuration interaction (CI) method. Due to the high numerical effort required, these methods are usually limited to small systems in practice. This can be attributed to the computational cost increase steeply with number of electrons. For instance, DFT, HartreeFock scale as $O\left(N^{3}\right)$ with system size; second-order Moller-Plesset perturbation theory (MP2) scales as $O\left(N^{5}\right)$; $\operatorname{CCSD}(\mathrm{T})$ scales as $O\left(N^{7}\right)$; and full $\mathrm{CI}$ scales as $O(N$ !). Here, cubic scaling means that, if the number of electrons in the system is doubled, the required computational time will increase by a factor of eight. There are ongoing efforts to reduce the scaling of quantum mechanical methods. A logical algorithmic progress would be linear-scaling or $O(N)$ method. In early 90 s, Weitao Yang developed the divide-and-conquer method based on locality and realized the first time a reduction to linear-scaling complexity in DFT calculations. This begins the research development in linear-scaling methods and several linear-scaling quantum mechanical methods are now in use that can deal with systems up to few thousand atoms.

Whereas linear-scaling methods has substantially extended their computational limit to few thousand atoms, researchers from different fields in material sciences, biology, nanoscience, environmental science are demanding computational methods that can address more complex and larger systems. In many fields, the frontier of decreasing sizes that can be probed by experiments meets that of increasing sizes amenable to simulations. These have fostered the development of multi-scale approach for simulations of more complex systems. In chemistry, a widely used multi-scale method, the quantum mechanics/ molecular mechanics (QM/MM) method, has become a popular tool for investigating chemical reactions in condensed phases. In QM/MM methods, the region of the system in which the chemical process takes place is treated by quantum mechanical method, while the environment is described by a molecular mechanics force field.

Within this approach, chemical reactivity can be studied in biological systems. In electronic engineering, the continuous miniaturization of semiconductor devices has reached the $22 \mathrm{~nm}$ node. In this scale, atomic features and quantum mechanical effects in highly scaled devices have become pronounced than ever before. An appropriate account of quantum effects in electronic device simulation is one critical factor in the semiconductor industry in the upcoming decades. Inspired by the success of $\mathrm{QM} / \mathrm{MM}$ method, a multiscale quantum mechanics/electromagnetics $(\mathrm{QM} / \mathrm{EM})$ method is developed to model and simulate the electric responses of electronic devices. The electronic device is partitioned into the active scattering region that requires quantum mechanical treatment and the passive region where a classical description is sufficiently accurate. As a result, the entire electronic device can be modeled efficiently with good accuracy. The method has been applied to a carbon nanotube (CNT) based electronic device embedded in a silicon block.

Advances in technology is often the key success in many industrial products, for example, the replacement of vacuum tube by silicon semiconductor, leading to today's new era of computer and information; development and application of nanotechnology also create a huge impact in the industry. Multi-scale simulation enables us to study and understand problems in different scale theoretically, starting from firstprinciples calculations. It can be applied to any simulation and design of engineering systems. This is the ultimate goal of the theoretical and computational science. To achieve this goal, the development of more efficient theoretical calculation methods, numerical algorithms and computer power are continuously pursued.

*Corresponding author: Yam Chi Yung, Beijing Computational Science Research Center, China, E-mail: yamcy@yangtze.hku.hk

Received March 26, 2013; Accepted March 26, 2013; Published April 01, 2013

Citation: Yung YC (2013) Multi-scale Simulations. Mod Chem appl 1: e107. doi:10.4172/mca.1000e107

Copyright: (c) 2013 Yung YC. This is an open-access article distributed under the terms of the Creative Commons Attribution License, which permits unrestricted use, distribution, and reproduction in any medium, provided the original author and source are credited. 